\title{
Automatic Thesaurus Generation through Multiple Filtering
}

\author{
Kyo KAGEURA ${ }^{\dagger}$, Keita TSUJI ${ }^{\ddagger}$, and Akiko, N. AIZAWA ${ }^{\dagger}$ \\ tNational Institute of Informatics \\ 2-1-2 Hitotsubashi, Chiyoda-ku, Tokyo, 101-8430 Japan \\ E-Mail: $\{$ kyo,akiko\}@nii.ac.jp \\ ${ }^{\ddagger}$ Graduate School of Education, University of Tokyo, \\ 7-3-1 Hongo, Bunkyo-ku, Tokyo, 113 Japan \\ E-Mail: i34188@m-unix.cc.u-tokyo.ac.jp
}

\begin{abstract}
,
In this paper, we propose a method of generating bilingual keyword clusters or thesauri from parallel or comparable bilingual corpora. The method combines morphological and lexical processing, bilingual word aligmment, and graph-theoretic cluster generation. An experimont shows that the method is promising.
\end{abstract}

\section{Introduction}

In this paper, we propose a method of automatic bilingual thesaurus generation by a combination of methods or multiple filtering. The procedure consists of three modules: (i) a morphological and lexical processing module, (ii) a translation pair extraction module, and (iii) a cluster generation module. The method takes parallel or comparable corpora as input, and produces as output bilingual keyword clusters with a reasonable computational cost.

Our aim is to construct domain-oriented bilingual thesauri, which are much in need both for cross-language IR and for technical translators. We assume domain-dependent parallel or comparable corpora as a source of information, which are abundant in case of Japanese and English.

The techniques used in each module are reasonably woll developed, including statistical word alignment methods. However, there remain at least three problems: (i) ambiguity of multiple hapax combinations in an alignment, which cannot be resolved by purely statistical methods, (ii) syntagmatic unit mismatches, especially in such cases as English and Japanese, and (iii) difficulty in final cleaning-up ${ }^{1}$.

In this paper, we slow that the proper combination of the above modules can be useful especially for resolving the cleaning-up problem and can produce good results in bilingual cluster or thesaurus generation.

\section{Method}

The procedure for thesaurus generation consists of the following three main modules.

(1) Morphological and lexical processing module: keyword units ${ }^{2}$ for English and Japanese are extracted separately.

(2) Translation pair extraction module: statistical weighting is applied to a corpus which has been through the morphological and lexical processing module. The aim of this stage is not to determine unique translation pairs, but to restrict translation candidates to a reasonable extent.

(3) Cleaning and cluster generation module: a bilingual keyword graph is constructed on the basis of the pairs extracted at translation pair extraction module, and a graph-theoretic method is applied to the keyword graph, to generate proper keyword clusters by removing erroneous links.

\footnotetext{
${ }^{1}$ If we want to obtain a clean lexicon, minor translation variations tend to be omitted, while many errors would be included if we want to retain minor variations.

${ }^{2}$ The word 'keyword' implies words that are important with respect to documents or domains. In this paper, we use the word for convenience, roughly in the same sense as "content-bearing words". If necessary, a module of keyword or tcrm weighting (e.g. Frantzi \& Ananiadou 1995; Nakagawa \& Mori 1998) can be incorporated easily.
} 


\subsection{Morphological \& lexical processing}

At this stage, basic lexical units or keyword candidates are extracted. We separately extract minimum or shortest units and maximum or longest complex units as syntagmatic units for keyword candidates. So two outputs are produced from this module, i.e. a bilingual keyword corpora of minimum units and another of maximum units.

The processing proceeds as follows:

(a) Morphological analysis

First, the corpus is morphologically analysed and POS-tagged. Currently, JUMAN3.5 (Kurohashi \& Nagao 1998) is used for Japanese and LT_POS/LT_CHUNK (Mikheev 1996) is used for English.

(b1) Extraction of minimum units

Minimum units in English are simply dofined as non-functional simple words extracted from the output of LT_POS. Minimum meaningful units in Japanese are defined as:

C_Prefix* (C_Adv|C_Adj|N) C_Suffix* where $C_{-}$indicates that the unit should consist of eithor Chinese characters or Katakana ${ }^{3}$.

(b2) Extraction of maximum units

Maximum complex units for English are the units extracted by LT_CHUNK, with some adhoc modifications.

Maximum complex units for Japanese are defined by the following basic pattern,

${ }^{-} C_{-} A d j *$ (C_Affix|C_Adv|C_Adj|N)+

where ${ }^{\circ} \mathrm{C}$ means that the unit should begin with either Chinese character or Katakana. The pattern remains deliberately coarse, to absorb errors by JUMAN. Coarse patterns with simple character type restrictions produce better results than grammatically well-defined syntagmatic patterns. A separate stop word list for affixes is also prepared together with an exceptional treatment routine, to make the Japanese. units better correspond to English units ${ }^{4}$.

After these processes, two corpora, one consisting of minimum units and the other of max-

\footnotetext{
${ }^{3}$ In addition, we have made a few ad-hoc rules to screen out some consistent errors produced by the morphological analysers.

${ }^{4}$ For instance, the Japanese suffix 'f fl' is climinated because it corresponds in most cases to the English word 'for', which tends to be excluded from chunks made by LT_CHUNK.
}

imum units, are created.

Intermediate constituent units are not extracted, because their inter-lingual unit correspondence is less reliable. Also, many important intermediate units of longer complex units appear themselves as an independent complex unit in a large domain-specific corpus, and, even if they do not, intermediate units can be extracted on the basis of minimum and maximum translation pairs if necessary.

\subsection{Extraction of translation candidates}

The module for extracting translation candidate pairs consists of statistical weighting and postprocessing. These are applied to the data of minimum units and maximum units separately. After that, the two data are merged to make input for the cluster generation module.

\section{(a) Statistical weighting}

Many methods of extracting lexical translation pairs have been proposed (Daille, Gaussier \& Langé 1994; Eijk 1993; Fung 1995; Gale \& Church 1991; Hiemstra 1996; Hull 1998; Kupiec 1993; Mclamed 1996; Smadja, McKcown \& Hatzivassiloglou 1996). Though it is difficult to evaluate the performance of existing methods as they use different corpora for evaluation ${ }^{5}$, the performance does not seem to be radically different. We adopted log-likelihood ratio (Danning 1993), which gave the best performance among crude non-itcrative methods in our test experiments ${ }^{6}$.

\section{(b) Postprocessing filter}

As the output of statistical weighting is simply a weighted list of all English and Japanese co-occurring pairs, it is necessary to restrict translation candidates so that they can be effectively used in the graph-theoretic cluster generation module. In addition to restricting possible translation pairs, it is necessary to determine unique translation pairs for hapax legomena. We use both macro- and micro-filtering heuristics to restrict translation candidates.

\footnotetext{
${ }^{5}$ A common testbed exists for French-English alignment (Veronis 1996-99) but not for Japanese-English.

${ }^{6}$ At the time of writing this paper, we have finished a preliminary comparative experiments of various methods, among which the method proposed by Melamed (1996) gave by far the best result. We are thus planning to replace this module with the method proposed by Melamed (1996).
} 
Two macro heuristics, applied to the overall list of pairs, are defined, i.e. (i) a proper translation should have a statistical score higher than the threshold $X_{S}$, and (ii) a keyword should have maximally $X_{C}$ translations or $X_{P} \times$ token frequency when the frequency is less than $X_{C}$.

Micro heuristics uses the information within each alignment; we assume that a keyword in one language only has one translation within an alignment ${ }^{7}$. Selecting unique pairs in each alignment is achieved by recursively taking a pair with the highest score within an alignment, each time deleting other pairs which have the same English or Japanese elements ${ }^{8}$.

After this process, the data of minimum units and maximum units are merged, which constitutes input for the next stage.

\subsection{Graph-theoretic cluster generation}

Up to this stage, the cooccurrence information used to extract pairs has the depth of only one. In order to climinate erroncous translations, we re-organise the extracted pairs into graph and use multi-level topological information by applying the graph-theoretic method. For explanation, let us assume that we obtained the data in Table 1 from the previous module as an input to this module.

Firstly, the initial keyword graph is constructed, where each node represents an English or Japanese keyword, and a link or edge represents the pairwise relation of corresponding keywords. We define the capacity or strength of a link by the frequency of occurrence of the pair in the corpus, i.e. the number of alignments in which the pair occurs ${ }^{9}$. Figure 1 shows the

\footnotetext{
${ }^{7}$ This is not true for longer alignment units such as full texts. However, this will apply to parallel titles and abstracts which are readily available. Many lexical alignment methods starting from sentence-level alignments assume this or some variations of this.

${ }^{8}$ Many maximum unit pairs in fact have the same score. We used the arithmetic mean of the constituent minimum units to resolve alignment, ambiguity.

${ }^{9}$ The score of likelihood ratio is a possible altcrnative for link capacity, but the result of a preliminary experiment was no better. In addition, after selecting pairs by threshold, whether a pair constitutes a proper translation or not is not a matter of weight, because threshold setting implies that all pairs above that are regarded as correct. So we adopt simple frequency as the link capacity. However, we notice a lack of affinity between the
}

\begin{tabular}{|c|c|c|}
\hline Japanese keywords & English keywords & frequency \\
\hline キーワード & information retricval & 1 \\
\hline キーワード & keyword & 39 \\
\hline テ打スト検索 & information retricval & 1 \\
\hline テキスト检䇠 & text retrieval & 6 \\
\hline テキス卜娭率 & text search & 3 \\
\hline 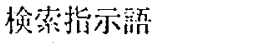 & keyword & 1 \\
\hline 広域情郝検沗 & information retrieval & 1 \\
\hline 情報檢案 & information gathering & 4 \\
\hline 情報检祭 & information retreival & 1 \\
\hline 情報検索 & information retrieval & 320 \\
\hline 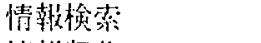 & information search & 5 \\
\hline 情赫则集 & information gathering & 6 \\
\hline 情報仪集 & information retrieval & 1 \\
\hline 文献检热 & bibliographic search & 1. \\
\hline 文献䐿率 & document retricval & 11 \\
\hline 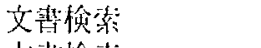 & document retrieval & 19 \\
\hline 文慧朲索 & text retrieval & 1 \\
\hline
\end{tabular}

Table 1. Input example for cluster generation

initial keyword graph made from the data in Table 1. The task now is to detcet and remove erroneous links to generate independent graphs or clusters consisting of proper translation pairs ${ }^{10}$.

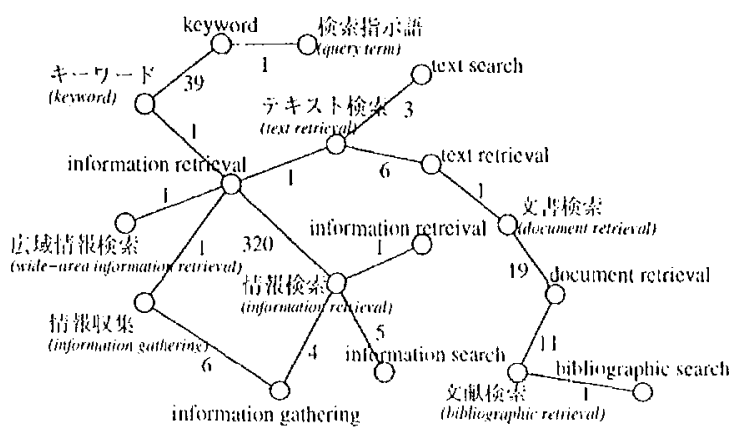

Figure 1. Example of initial keyword graph

The detection algorithm is based on the simple principle that sets of links, which decompose a connected keyword cluster into disjoint subclusters when they are removed from the original cluster, are candidates for improper translations. In graph theory, such a link set is called an edge cut and the edge cut with the minimal total capacity is called a minimum edge cut. A minimum edge cut does not necessarily imply a single translation crror. An efficient al-

statistical alignment method we used here and the definition of link capacity, which is currently under examination and will be improved by renewing the alignment module.

${ }^{10}$ This approach is radically different from statistically oriented word clustering (Decrwester et. al 1990; Finch 1993; Grefenstette 1994; Schütze \& Pedersen 1997; Strzalkowski 1994); this is why we use the word 'cluster generation' instead of 'clustering'. 


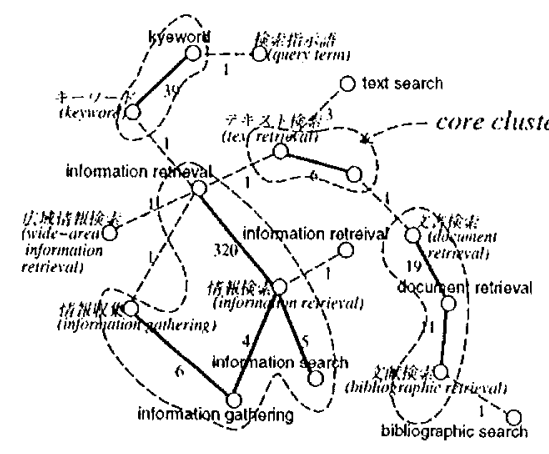

(a)

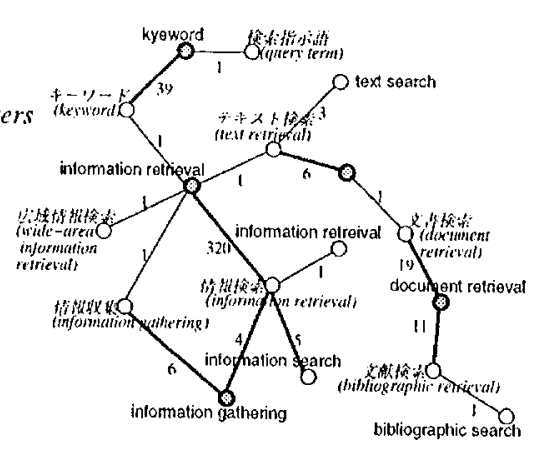

(b)

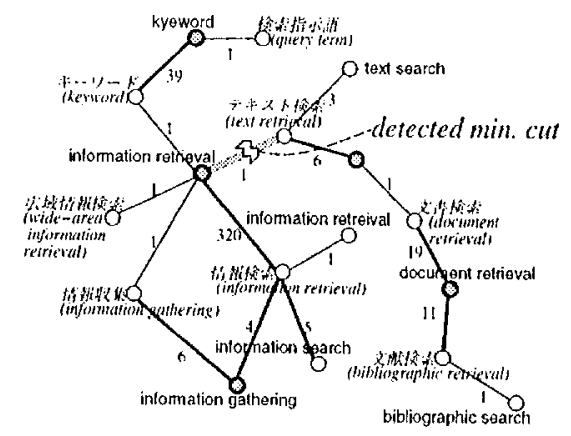

(c)

Figure 2. Steps of graph-theoretic cluster generation

gorithm exists for minimum edge cut detection (Nagamochi 1993).

Our procedure first checks links that should not be climinated, using the conditions: (i) the frequency is no less than $N_{\alpha}$, (ii) the Japanese and English notations are identical, or (iii) either of the Japanese or English expressions have only one corresponding translation (Figurc 2 (a); it is assumed that $N_{\alpha}=N_{\beta}=N \epsilon=3$ ). Secondly, core keywords whose frequency is no less than $N_{\beta}$ are checked (Figure $2(\mathrm{~b})$ )). This is used for the restriction that each cluster should include at least one core keyword. Lastly, edge cuts with a total capacity of less than $N_{\epsilon}$ are detected and removed (Figure 2 (c)). This procedure is repeated recursively until no further application is possible. Figure 3 shows the state after these steps are applied.

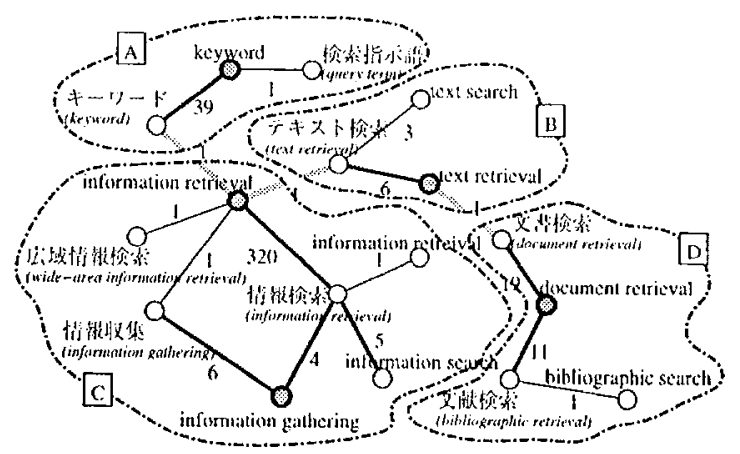

Figure 3. Generated clusters

\section{Experiment}

\subsection{Settings and procedures}

We applied the method to Japanese and English bilingual parallel corpus consisting of 25534 title pairs in the field of computer sci- ence. Table 2 shows the basic quantitative information after morphological and lexical filtering was applied.

\begin{tabular}{lllll}
\hline \multicolumn{4}{c}{ Minimum units } \\
\hline Japanese & Token: 178091 & Type: & 14938 \\
English & Token: & 154554 & Type: & 12634 \\
\hline \multicolumn{4}{c}{ Maximum units } \\
\hline Japanese & Token: & 89742 & Type: & 38813 \\
English & Token: & 80018 & Type: & 41693 \\
\hline
\end{tabular}

Table 2. Basic quantity of the data

In the pair extraction module, the threshold $X_{S}$ was set to $10^{11}$. The parameter $X_{C}$ was set to 10 and $X_{P}$ to 0.5 . As a result, 28905 translation candidate pairs were obtained, with 24855 Japanese and 23430 English keywords. Of these, 20071 pairs occurred only once and 3581 only twice. The most frequent pair occurred 3196 times in the corpus. 8242 (28.5\%) were minimum unit pairs, and 20663 (71.5\%) were maximum unit pairs.

Table 3 shows the number of keywords which had $N$ translations. On average, a Japanese keyword had 1.16 English translations, while an English keyword had 1.23 Japanese translations.

\begin{tabular}{rrr|rrr}
\hline$N$ & Jap. & Eng. & $N$ & Jap. & En. \\
\hline 1 & 21796 & 19778 & 5 & 62 & 157 \\
2 & 2409 & 2693 & 6 & 10 & 59 \\
3 & 412 & 437 & 7 & 7 & 17 \\
4 & 159 & 285 & 8 & 0 & 4 \\
\hline \multicolumn{4}{c}{ Table 3. Number of translations }
\end{tabular}

${ }^{11}$ This is purely heuristic. Minimum units and maximum units are given different scores. But only 3 pairs below this threshold were proper translation pairs in 100 random samples of minimum unit pairs, and 5 in 100 samples of maximum units. 
Evaluating recall and precision on the basis of 100 randomly selected title pairs, which consisted of 778 keyword token pairs, the precision tokenwise was $84.06 \%$ (654 correct translations) and the recall was $87.08 \%$ (654 of 751 correct pairs). Typewise precision was $81.65 \%$ (543 correct of 665 pairs).

The initial keyword graph generated from these 28905 translation candidates consisted of 19527 independent subgraphs, with the largest cluster conlaining $270 \mathrm{~L}$ pairs (i.e. $9.3 \%$ of all the pairs). The cluster generation method was applied with parameters $N_{\alpha}=4, N \epsilon=10$ and $N_{\beta}=1^{12}$. As a result, 893 translation pairs were removed, and 20357 bilingual clus. ters were generated. The maximum cluster now contained only 64 pairs. 'lable 4 shows the number of chusters by size given by number of pairs.

\begin{tabular}{rr|rr}
\hline size & no. of clusters & size & no. of clusters \\
\hline 1 & 16693 & $5-9$ & 322 \\
2 & 2354 & $10-19$ & 52 \\
3 & 504 & $20-64$ & 22 \\
4 & 410 & & \\
\hline \multicolumn{3}{r}{ Table } & 4. Number of clusters by sizo
\end{tabular}

\subsection{Overall evaluation}

The result was manually cvaluated from two points of view, i.e. consistency of clusters and correctness of link removal ${ }^{13}$.

(1) To check the internal consistency, clusters wore classified into three groups by size, and were separately evaluated. 2000 'small' clusters, consisting of only one pair, were randomly sampled and evaluated as 'correct' (c), 'more or less correct' (m) or 'wrong' (w). 400 medium size clusters consisting of $2-9$ pairs and all the 74 large clusters consisting of 10 or more pairs were evaluated as 'consistent' (c: consisting only of closely related keywords), 'mostly consistent' (m: consisting mostly of related keywords), 'hybrid' (h: consisting of two or more different keyword groups: h) or 'bad' (w'). 'lable 5 shows the result of the evaluation. The general performance is very good, with more or less $80 \%$ of the clusters being meaningful.

\footnotetext{
12. This is again determined heuristically. For an exanination of the effect of parameters, see Airawa \& Kageura (to appear).

13 'The evaluation was done by the first author. Currently no cross-checking has been carried out.
}

For small clusters, the porformance was separately evaluated for minimum and maximum unit pairs. Note that the ratio of maximum unit pairs is comparatively higher in the small cluster than the overall average. Most pairs evaluated as partially correct, as woll as some wrong pairs, suffered from mismatch of the syntagmatic units.

\begin{tabular}{|c|c|c|c|c|}
\hline & $c$ & $\mathrm{~m}$ & $\mathrm{w}$ & totial \\
\hline \multirow[t]{2}{*}{ Small } & 1389 & 370 & 241 & 2000 \\
\hline & $(69.5 \%)$ & $(18.5 \%)$ & $(12.1 \%)$ & $(100 \%)$ \\
\hline \multirow{2}{*}{ minimum } & 288 & 26 & 69 & 383 \\
\hline & $(75.2 \%)$ & $(6.8 \%)$ & $(18.0 \%)$ & $19.2 \%$ \\
\hline \multirow[t]{2}{*}{ maximum } & 1101 & 344 & 172 & 1617 \\
\hline & $(68.1 \%)$ & $(21.3 \%)$ & $(10.6 \%)$ & $80.9 \%$ \\
\hline & c & $\mathrm{m}$ & h & W \\
\hline \multirow[t]{2}{*}{ Medium } & 116 & 148 & 32 & 104 \\
\hline & $(29.0 \%)$ & $(37.0 \%)$ & $(8.0 \%)$ & $(26.0 \%)$ \\
\hline Large & $\begin{array}{r}8 \\
(10.8 \%)\end{array}$ & $\begin{array}{r}18 \\
(24.3 \%)\end{array}$ & $\begin{array}{r}43 \\
(58.1 \%)\end{array}$ & $\begin{array}{r}5 \\
(6.8 \%)\end{array}$ \\
\hline
\end{tabular}

Table 5. Tivaluation of internal consistency

$73 \%$ of the medium sized clusters were ' $c o r-$ rect', 'mostly correct' or 'hylorid'. Among the 'mostly correct' and 'hybrid' clusters, 97 (91 and 6 respectively) were mainly caused by the mismatch of the units. For instance, in the case: $\{$ 岸邀，最適化，岸適大，optimization，optimal, optimisation, oplimum, network optimization \}, the last English keyword has the excess unit 'network'. Other 'mostly correct' and 'tyybrid' clusters were due to the problem of corpus Srequencies.

Among the large clusters, more than half were 'hybrid'14. Among the 'mostly correct' and 'hybrid' large clusters, only $8(3+5)$ were due to unit mismatch, while $53(15+38)$ were due to quantilative factors. This shows a striking contrast to the medium sized clusters. Large hybrid clusters tended to include many common word pairs which occur frequently. For instance,

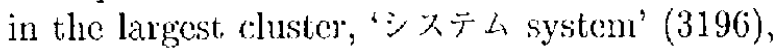
'北発 development' (1097), '酸济 design' (1073), and '環境 enviromment' (890) are included due to indirect associations. The following are two examples of hybrid clusters, whose hybridness comes from quantitative factors and unit mismatches respectively:

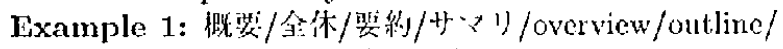
summary/summarization/overall

\footnotetext{
14 And most of the sub-clusters in these hybrid clusters are 'mostly correct'.
} 
Example 2: パターン/パタン/パターン諗裁/照合 /バーンマッホング/ハターン照合法/ハターン照命 /pattern/patterns/patten/patterm matching

In the first case, the 'overall' group and the 'summary' group are mixed up. In the second case, the mismatch of syntagmatic units is caused by borrowed words. In fact, many errors caused by the mismatch of syntagmatic units involve borrowed words written in Katakana.

(2) To look at the performance of graphtheoretic cluster generation, we examined tho removed pairs from two points of vicw, i.e. the correctness of link removal and the internal consistency of clusters generated by link removal. For the former, we introduced three categories for evaluation: mismatched pairs correctly removed (c), proper translation pairs wrongly removed ( $w$ ), and pairs of related meaning removed (p). The consistency of newly generated clusters were evaluated in the same manner as above.

\begin{tabular}{lrrrr}
\hline & $c$ & $\mathrm{p}$ & $\mathrm{w}$ & total \\
\hline $\mathrm{cc}$ & $90(10.1)$ & $53(5.9)$ & $39(4.4)$ & $182(20.4)$ \\
$\mathrm{cm}$ & $148(16.6)$ & $56(6.6)$ & $32(3.6)$ & $236(26.4)$ \\
$\mathrm{ch}$ & $96(10.8)$ & $20(2.2)$ & $6(0.7)$ & $122(13.7)$ \\
$\mathrm{mm}$ & $44(4.9)$ & $29(3.3)$ & $30(3.4)$ & $103(11.5)$ \\
$\mathrm{mh}$ & $52(5.8)$ & $13(1.5)$ & $5(0.6)$ & $70(7.8)$ \\
$\mathrm{hh}$ & $30(3.4)$ & $3(0.3)$ & $3(0.3)$ & $36(4.0)$ \\
\hline $\mathrm{xc}$ & $42(4.7)$ & $9(1.0)$ & $9(1.0)$ & $60(6.7)$ \\
$\mathrm{xm}$ & $28(3.1)$ & $8(0.9)$ & $20(2.2)$ & $56(6.3)$ \\
$\mathrm{xh}$ & $8(0.9)$ & $2(0.3)$ & $5(0.6)$ & $15(1.7)$ \\
$\mathrm{xx}$ & $4(0.5)$ & $1(0.1)$ & $8(0.9)$ & $13(1.5)$ \\
\hline all & $542(60.7)$ & $194(21.7)$ & $157(17.6)$ & $893(100)$ \\
\hline
\end{tabular}

Table 6 . Evaluation of removed links

Table 6 shows the result of evaluation of all the 893 removed pairs. ' $c$ ' ' $p$ ' and ' $w$ ' in the top row indicate types of removed links, and ' $c c$ ', 'cm' etc. in tho lefumost column indicate internal consistencies of two clusters generated by link removal. A total of $157(17.6 \%)$ of the removed links were correct links wrongly romoved, but among them, 115 links did not produce 'bad' clusters. If we consider them to be tolerable, only 42 removals ( $4.7 \%$ ) were fatal errors.

By examining the removed links, we found that the links removed at the higher edge capacity included more wrongly removed pairs. For instance, among 142 edges removed at capacity 4 (which is the maximum deletable value sot by $N_{\alpha}$ ), 41 or $28.9 \%$ were wrongly removed correct translations, while among 288 links removed at capacity 1, only 15 or $5.2 \%$ were correct translations.

\section{Discussion}

From the experiment, we have found some factors that affect performance.

(1) Many errors were produced at the stage of extracting keyword units, by syntagmatic mismatch. A substantial number of them involved Japanese Katakana keywords. Therefore, in addition to the general refinement of the morphological processing module, the performance will be improved if we use string proximity information to determine syntagmatic units ${ }^{15}$.

(2) We expect that some crrors produced by statistical weighting and filtering could bo removed by applying stemming and orthographic normalisations, which are not fully exploited in the current implementation. Looking back from the cluster generation stage, frequently occurring keywords tend to cause problems due to indirect associations. At the time of writing, we are radically changing the statistical alignment module based on Melamed (1996) and incorporating iterative alignment anchoring routine so that the method can be applied not only to titles but also to abstracts, ctc. Used in conjunction with string proximity and stemming information, we might be able to retain minor variations properly.

(3) At the cluster generation stage, we observed that correct links tend to be wrongly removed for ligher capacities of edge cut. In the current implementation, the parameter values remain the same for all the clusters. Performance will be improved by introducing a method of dynamically changing the parameter values according to the cluster size and the frequencies of their constituent pairs.

\section{Conclusion}

We have proposed a method of constructing bilingual thesauri automatically, from parallel or comparable corpora. The experiment showed that the performance is fairly good. We are currently improving the method further, along the lines discussed in the previous section. Further experiments are currently being carried out, using the data of narrower domains (e.g. artificial

\footnotetext{
${ }^{15}$ This can also be used for resolving hapax ambiguity.
} 
intelligence) as well as abstracts instcad of titiles.

At the next stage, wo are plaming to evaluate the method from the point of view of performance of gonerated clusters in practical applications. We are currently planning to apply the generated clusters to query expansion and user navigation in cross-lingual H, as well as to on-line dictionary lookup systems used as translation aids.

\section{Acknowledgement}

This rescarch is a part of the research project " $\Lambda$ Study on Ubiquitous Information Systems for Utilization of Highly Distributed Information Resources", lunded by the Japar Society for the Promotion of Science.

\section{References}

[1] Ainawa, A. N. and Kageura, K. (to appear) "A graph-based approach to tho nutomatic goneration of multilingual keyword chusters." In: Bouligault, D., Jacquemin, C. and l'Homme, M-C. (eds.) Recent Advances in Computational Terminology. Amsterdam: Jolm Benjarnins.

[2] Dagan, I. and Church, K. (1994) "Temight: Identifying and translating technical terminology. "Proce of the Fourth ANIS. p.34-40.

[3] Daille, B., Gaussier, E. and Langé, J. M. (1994) "Towards automatic extraction of monolingual and bilingual terminology." COLING'Y4. p. $515 \cdot 521$

[4] Decrwester, S., Dumais, S. T., lunnas, G. W., Landater, T. K. and Harshman, R. (1990) "Indexing by latent semantic analysis." JASIS. $41(6)$, p. $391-407$.

[5] Dunning, T. (1993) "Accurate methods for the statistics of surprise and coincidence." Computational Linguistics. 19(1), p. 61-74.

[6] Eijk, van dor P'. (1993) "Automating the acquisition of bilingual terminology." Proc. of the 6th FACL. p. 113-119.

[7] Finch, S. P. (1993) Finding Structure in Janguage. PhD Thesis. Edinboungh: University of Frdinbourgh.

[8] Frantzi, K. T. and Ananiadou, S. (1995) "Statistical measures for teminological extraction." Proc. of 3rd Int'l Conf. on Statistical Analysis of 'T'extual Data. p. 297-308.

[9] Fung, P. (1995) "A pattern matching method for finding noun and proper noun translations from noisy parallel corpora." Proc. of 33rd ACI. p. 233-236.
[10] Gale, W. A. and Church, K. W. (1991) "Identifying word correspondences in parallel texts." Proc. of DARPA Speech and Netural Language. Workshop. 1) 152-157.

[11] Grefenstette, G. (1994) Explorations in Automatic Thesaurus Discovery. Boston: Kluwer Acarlemic.

[12] Hiemstra, D. (1996) Using Statistical Methods to Creat a Bilinqual Dictionary. MSc Thesis, Twente University.

[13] Hull, D. A. (1998) "A practical approach to terminology alignment." Computerm'08. p. 1 7 .

[14] Kitamura, M. and Matsumoto, Y. (1997) "Automatic Extraction of Translation Patterns in Parallel Corpora." Transactions of IPSJ. 38(4), p. 727.735 .

[15] Kupiec, J. (1993) "An algorithm for finding noun phrase correspondences in bilingual corpora." Proc of 31 st ACL. p.17-22.

[16] Kurohashi, S. and Nagao, M. (1998) Japanese Morphological Analysis System Juman version 3.5 User's Manual. Kyoto: Kyoto University.

17] Molamed, I. D. (1996) "Automatic construction of clean broad-coverage translation lexicons." 2nd Conference of the Association for Machine Translation in the Americas. p. 125 -134.

[18] Mikheev, A. (1996) "Learning part-of-specch guessing rules from lexicon." COLING'96, p. $770-775$.

[19] Naganochi, II. (1993) "Minimum cut in a graph." In: liujisige, S. (od.) Discrete Structure and Algorithms II (Chapter 4). Tokyo: Kindaikagakusha.

[20] Nakagawa, H. and Mori, 'I'. (1998) "Nested collocation and compound noun for term extraction." Computerm'98. p. 6470.

[21] Schïtze, H. and Pedersen, J.O. (1997) "A cooccurrence-based thesaurus and two applications to information retrieval." Information Processing and Management. 33(3), p.307-318.

[22] Smadja, F., McKcown, K. R. and Hatzivassiloglou, V. (1996) "Lranslating collocations for bilingual lexicons: $\Lambda$ statistical approach." Computational Linguistics. 22(1), p. 1--38.

[23] Str\%alkowski, 'I'. (1994) "Building a lexical domain map from text corpora." COLING:94, p.604-610.

[24] Veronis, .J. (1996-) "ARCADE: Evaluation of parallel text, alignment systems." http://www.lpl.univ-aix.fr/projects/arcade/

[25] Yonczawa, K. and Matsumoto, Y. (1998) "Zoshinteki taiouzuke ni yoru taiyaku tekisuto lara no hon'yaku hyougen no cyusyutu." Proc of the 4th Annual Mceting of the Association. for $N L Y^{\prime}$. p. 576-579. 